\title{
Bilan de la campagne de collecte des objets au radium à usage médical
}

\author{
J.P. PIERRE ${ }^{1}$, J.P. VIDAL ${ }^{\text {, }}$, J.C. MARTIN ${ }^{\prime}$, J.L. PASQUIER ${ }^{1}$
}

(Manuscrit reģu le 23 août 2001, accepté le 20 janvier 2002)

RÉSUMÉ Sur demande du ministre chargé de la Santé, l'OPRI et l'ANDRA lancent le $1^{\text {er }}$ décembre 1999 une campagne de collecte d'objets au radium autrefois utilisés en curiethérapie. Cette campagne répond à un souci de santé publique, compte tenu de réels risques pour des personnes pouvant manipuler ces objets sans précaution. En outre, son bien fondé s'est renforcé, ces dernières années, par de nombreuses affaires de découvertes d'objets au radium en déshérence. Cette collecte est basée sur le volontariat des détenteurs qui signalent la présence d'objets au radium dans leurs locaux auprès d'une cellule opérationnelle OPKI-ANDRA, au moyen d'un numéro vert. Cette cellule analyse chaque demande et apprécie le degré d'urgence de la situation. La priorité est accordée aux particuliers qui ne disposent pas de conditions d'entreposage satisfaisantes sur le plan de la radioprotection. Les équipes de l'OPRI effectuent les collectes suivant un protocole strict garantissant leur sécurité, le bon déroulement des opérations et le respect des règles du transport de matières radioactives. Les 90 interventions réalisées ont permis de collecter 517 objets représentant une activité de $1,32 \times 10^{11} \mathrm{~Bq}$. Une fois conditionnés, ces objets ont été acheminés au CEA de Saclay, pour un entreposage en toute sécurité en attendant le stockage définitif par l'ANDRA.

ABSTRACT Campaign to gather medical devices containing radium: results.

On December 1, 1999, at the request of the French Health Ministry, OPRI and ANDRA launched a campaign to gather medical devices containing radium, formerly used in brachytherapy. This campaign addressed a public health issue because of the risks actually involved in a careless handling of these objects. Moreover the growing number of reported scattered radium medical devices in the last few years reinforced the necessity of the campaign. The gathering was initiated by a call of the owners (hospitals, caring centers, retired doctors or their heirs) to a toll free number. OPRI or ANDRA then appreciated the situation urgency. Priority was given to private people because most of them did not have suitable storage facilities. OPRI teams operated according a strict protocol guaranteeing their own safety, proper procedures and compliance with transport regulations for radioactive materials. 517 objects amounting to an activity of $1.32 \times 10^{11} \mathrm{~Bq}$ have been gathered in 90 operations. Properly packaged they were transported to and safely stored at the CEA Saclay site before their permanent storage in the ANDRA facilities.

\section{Introduction}

À la demande du ministère chargé de la Santé, l'OPRI et l'ANDRA ont lancé le $1^{\text {er }}$ décembre 1999 pour une durée d'un an, une campagne nationale de collecte des

${ }^{1}$ Office de protection contrc les rayonnements ionisants, BP 35, 78116 le Vésinet Cedex. France. 
objets au radium à usage médical, utilisés dans la première partie du $\mathrm{XX}^{\mathrm{e}}$ siècle par les médecins en curiethérapie. Cette campagne répond à un souci de santé publique, compte tenu des réels risques pour les personnes pouvant manipuler ces objets sans précaution. En outre, le bien fondé de cette campagne s'est trouvé renforcé par la multiplication, ces dernières années d'affaires liées à la découverte de sources de radium en déshérence. De plus, de nombreuses demandes de récupération provenant des détenteurs n'avaient pas été satisfaites, faute d'une procédure adaptée, bien que le SCPRI ait pris l'initiative au cours des années 80 de procéder à des opérations de récupération. L'expérience a montré que les risques liés à leur détention sont bien souvent méconnus des détenteurs mais aussi sous-estimés des anciens utilisateurs.

Cet article dresse le bilan d'une année de collecte réalisée par les équipes d'intervention de l'OPRI (en complément de leurs missions de contrôles destinées à assurer la veille sanitaire de la population en matière de radioprotection) dans des conditions organisationnelles et techniques dont la gestion présente souvent quelques difficultés. Il constitue une base de retour d'expérience de la radioprotection opérationnelle appliquée dans le domaine public au sein de structures différentes allant du centre de soins jusque chez le particulier.

\section{De la découverte de la radioactivité jusqu'à l'utilisation du radium (ANDRA, 1997 ; Boudia, 1997)}

En disposant par hasard une plaque photographique sur des sels d'uranium, le physicien Henri Becquerel découvre en 1896, la radioactivité naturelle. Deux années plus tard, Pierre et Marie Curie découvrent en 1898 le polonium et le radium. En 1903, tous les trois reçoivent le prix Nobel de Chimie.

En se rendant à une conférence à Londres, Henri Becquerel transporte une source de radium empruntée à Pierre Curie dans son gilet, et constate par la suite, l'apparition d'une tache rouge sur sa peau. En 1901, Pierre Curie renouvelle l'expérience sur son bras. Les Curie envisagent alors une application médicale des sources de rayonnement. Dès 1901, ils mettent à la disposition des hôpitaux des sources de radium. Les premiers résultats sont incohérents et contradictoires, car par manque de repère dosimétrique, l'effet des rayonnements est confondu avec l'effet placebo (appellation actuelle de l'amélioration que ressent un malade à la seule idée qu'il bénéficie d'un médicament prestigieux).

Ainsi, dès le début du $\mathrm{XX}^{\mathrm{e}}$ siècle, l'industrialisation de l'extraction du radium se développe et un certain nombre de laboratoires et d'usines se construisent, en particulier en région parisienne. Les plus connues, en raison des affaires récentes sont la Société nouvelle du radium, créée en 1907 par Jacques Danne, associé de 
Marie Curie, à Gif-sur-Yvette dans l'Essonne et l'usine de Nogent-sur-Marne construite par Armet de-Lisle sur le site de laquelle fut construite dans les années soixante une école aujourd'hui fermée.

Le processus d'extraction du radium à partir du minerai de pechblende nécessite de grandes quantités de matière première et de longs mois de traitement et de cristallisation en laboratoire : ainsi pour obtenir 1 à $2 \mathrm{mg}$ de bromure de radium, il faut une tonne de pechblende, cinq tonnes de produits chimiques et cinquante tonnes d'eau de lavage.

Les sels de radium sont donc très difficiles à obtenir et comme il existe une très forte disproportion entre l'offre et la demande, on assiste à une flambée des prix entre les années 1904 et 1913. De ce fait, le gramme de bromure de radium se négociait à $400000 \mathrm{~F}$ de l'époque et le gramme de radium pur à $750000 \mathrm{~F}$, ce qui représentait 200000 fois le prix de l'or.

Dès 1906, les médecins se rendent compte de la radiosensibilité des cellules cancéreuses. Ils y voient une opportunité dans le traitement des cancers de la peau et de tumeurs profondes. De nombreux médecins acquièrent alors des aiguilles de radium. En 1908, les affections cutanées sont traitées avec succès par radiumthérapie.

La Grande Guerre offre à Marie Curie la possibilité d'utiliser ce radioélément notamment dans la stérilisation et la cicatrisation des plaies.

Entre 1920 et 1930 , le radium connaît un fort engouement de la population française. La société entre dans les années folles du radium. De nombreux objets au radium dont l'utilisation de nos jours ferait pâlir le monde de la radioprotection ont été commercialisés dans le grand public : émanateurs au radium (ou fontaines à radon), laine pour layette, compléments pour engrais, bougies automobiles, aliments pour bétail, crèmes cosmétiques, compresses... À cette époque, le radium est paré de diverses vertus qu'il ne possède pas forcément. Par ailleurs, ce phénomène est facilité par une entrée des Belges sur le marché du radium qui par l'intermédiaire des mines du Haut-Katanga ont réussi à casser les prix.

En 1928, le Congrès international de radiologie médicale (CIRM) confie à la Commission internationale de protection radiologique (CIPR) la mission de concevoir un système de norme de protection contre les effets des rayons $\mathrm{X}$ et du radium, afin d'enrayer les accidents survenus aux radiologues militaires de la $\mathrm{I}^{\text {re }}$ Guerre mondiale.

Dans les années 30, les scientifiques commencent à distinguer les bienfaits thérapeutiques des rayonnements ionisants et leurs dangers. En 1934, Irène 
TABLEAU I

Les objets au radium à usage médical.

Medical objects containing radium.

\begin{tabular}{|c|c|c|c|c|}
\hline How & (1) & Composition & 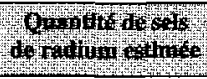 & 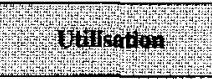 \\
\hline Aiguille & $\begin{array}{c}\phi_{\mathrm{ext}}=1,6 \mathrm{~mm} \\
\oint_{\mathrm{int}}=0,7 \mathrm{mtht} \\
L=20 \text { a } 50 \mathrm{~mm}\end{array}$ & $\begin{array}{l}\text { Corps cylindrique creux } \\
\text { en platine ou en or }\end{array}$ & Entre I et $5 \mathrm{mg}$ & $\begin{array}{l}\text { Implantation directe } \\
\text { sous fa peau }\end{array}$ \\
\hline Tube & $\begin{array}{c}\phi_{\mathrm{cxt}}=2,6 \text { à } 3 \mathrm{~mm} \\
\phi_{\mathrm{int}}=0,6 \text { à } 1 \mathrm{~mm} \\
L \cong 20 \mathrm{~mm}\end{array}$ & $\begin{array}{l}\text { Petit cylindre creux en } \\
\text { platine ou or }\end{array}$ & $5,10,15$ ou $20 \mathrm{mg}$ & $\begin{array}{l}\text { Curiethérapie } \\
\text { de surface }\end{array}$ \\
\hline Gaine & Epaisseur paroi $=1 \mathrm{~mm}$ & $\begin{array}{l}\text { Surconteneur recevant } \\
\text { aiguilles ou tubes }\end{array}$ & Jusqu'à $250 \mathrm{mg}$ & Idem tube \\
\hline $\begin{array}{l}\text { Plaque de } \\
\text { Monel }\end{array}$ & Entre 1 et $10 \mathrm{~cm}^{2}$ & $\begin{array}{l}\text { Support acier recouvert } \\
\text { d'une surface émaillée }\end{array}$ & $\begin{array}{l}5 \text { à } 20 \mathrm{mg} \\
(40 \mathrm{mg})\end{array}$ & Dermatologie \\
\hline Applicateurs & Variables & Pièces métalliques & $\begin{array}{l}\text { Potentiellement } \\
\text { contamines }\end{array}$ & $\begin{array}{l}\text { Facifter l'utilisation } \\
\text { des antres ORUM }\end{array}$ \\
\hline $\begin{array}{c}\text { Sonde } \\
\text { de Crowe }\end{array}$ & 20 à $30 \mathrm{~cm}$ de long & $\begin{array}{l}\text { Tige métallique : une } \\
\text { spatule à une extrémité, } \\
\text { la source à l'autre bout }\end{array}$ & $50 \mathrm{mg}(100 \mathrm{mg})$ & Application en ORL \\
\hline
\end{tabular}

et Frédéric Joliot-Curie découvrent la radioactivité artificielle alors que Marie Curie décède d'une leucémie aiguë cette même année. Cette découverte ouvre ainsi de nouvelles perspectives dans les applications médicales des rayonnements ionisants, notamment avec le cobalt radioactif et la radiothérapie métabolique.

Aujourd'hui le radium n'est plus utilisé à des fins médicales, puisqu'il a été remplacé par des radioéléments artificiels à vie courte. Cependant, certains médecins déclarent l'avoir employé jusque dans les années 90.

\section{Les différents objets au radium à usage médical (ORUM)}

Le tableau I présente un inventaire non exhaustif des principaux objets utilisés en curiethérapie. Ces données proviennent pour partie de la littérature (ANDRA, 1998 ; Dutreix et al., 1982 ; Tubiana, 1999) et des mesures réalisées par l'OPRI. À ce sujet, il convient de souligner que l'activité est estimée à partir d'une mesure de débit de dose absorbée. Dans ces conditions elle peut être surestimée car elle inclut également celle des descendants du radon qui se sont accumulés dans l'objet si son enveloppe est toujours étanche.

Dans le meilleur des cas, ces objets sont conditionnés dans de petits containers de plomb de faible épaisseur ( 1 à $2 \mathrm{~cm}$ ), qui peuvent eux-mêmes être disposés dans des boîtes en bois ou en inox. Très souvent, les containers de plomb sont sous-dimensionnés sous l'angle de la radioprotection par rapport à leur contenu, 
mais il arrive de rencontrer des situations dans lesquelles les objets ont été conditionnés dans des emballages de plus forte épaisseur. Les sondes de Crowe sont par contre protégées dans des emballages de plomb plus épais, en raison de leur activité.

\section{La première campagne de collecte des ORUM}

Des stocks d'ORUM inutilisés étaient détenus dans les hôpitaux et divers centres de soins, parfois dans des conditions de stockage ne garantissant pas le respect des règles de radioprotection. Dans les années 80 , le SCPRI avait organisé, une première campagne de collecte durant une dizaine d'années (de 1983 à 1993).

À l'issue de cette campagne, une centaine d'opérations de collecte ont été effectuées. Au total, ce sont près de $25 \mathrm{~g}$ de radium ${ }^{2}$, soit une activité de 25 Curies $(0,9 \mathrm{TBq})$ et 2800 objets (937 tubes, 29 gaines, 1840 aiguilles, 7 plaques de Monel, 11 sondes de Crowe) qui ont été collectés et mis en sécurité.

En l'absence de toute comptabilité précise de la quantité totale de radium produite et utilisée en France depuis le début du $X^{\mathfrak{e}}$ siècle, on estime que 100 à $150 \mathrm{~g}$ ont été mis en circulation, répartis de la manière suivante :

- entre 75 et $100 \mathrm{~g}$ dans les applications médicales sous forme de sources scellées (aiguilles, tubes, applicateurs, à hauteur de $70 \%$ ) et en cosmétologie sous forme de sources non scellées (pommade, poudre, crème, à hauteur de $30 \%$ ) ;

- entre 25 et $50 \mathrm{~g}$ dans les pratiques industrielles ou assimilées.

Au total, il est possible de penser qu'il reste à récupérer sous forme de sources scellées dans le domaine médical entre 5 et $20 \mathrm{~g}$ et entre 10 et $25 \mathrm{~g}$ dans le domaine industriel, le radium utilisé en cosmétologie, n'étant par hypothèse pas récupérable.

\section{Les détenteurs potentiels des ORUM}

Les premiers détenteurs sont bien entendu les médecins qui les utilisaient lors de leurs activités de soins. Certains d'entre eux encore en activité peuvent détenir ces objets sans pour autant les utiliser ou bien ne plus exercer la médecine, tout en les détenant toujours chez eux. Dans certains cas, ces médecins sont de nos jours décédés et la plupart du temps ils ont laissé leurs objets à leurs héritiers ou à leurs épouses.

\footnotetext{
${ }^{2}$ Quantité estimée par le SCPRI sur lá base des déclarations des détenteurs. Les estimations faites par le CEA montrent que l'activité collectée se situerait en fait uutour de $50 \mathrm{~g}$.
} 
Par ailleurs, ces médecins exerçaient soit dans leur propre cabinet, soit dans un centre de soins (hôpitaux civils ou militaires, cliniques, centres anticancéreux, centres de radiologie). Ces centres ont pu aussi dans le passé recueillir des sources détenues par des particuliers qui les ont apportées pour s'en débarrasser. Par conséquent, ils peuvent posséder aujourd'hui dans leurs locaux des objets, qui hormis un intérêt historique, n'ont plus d'applications médicales.

En outre, en raison de sa forte valeur marchande, le radium a fait l'objet de spéculations financières. Ainsi des notaires s'en sont procurés à des seules fins de placement. De ce fait, les notaires sont détenteurs potentiels. De plus, des particuliers, toujours à des fins spéculatives, ont pu en cacher dans des coffres de banque.

\section{Les risques liés à la détention d'objets au radium}

\subsection{Les caractéristiques du radium 226}

Le radium 226 est issu de la chaîne naturelle de l'uranium 238. Sa période radioactive est de 1600 ans. Il se désintègre par émission $\alpha(\mathrm{E} \alpha \mathrm{l}=4,6 \mathrm{MeV}$ à $5,5 \%$ et $\mathrm{E} \alpha 2=4,7 \mathrm{MeV}$ à $94,4 \%$ ) et donne un gaz radioactif, le radon 222 . Par une succession de désintégrations $\alpha$ et $\beta$ le radon 222 ( 3,8 jours de période) donne naissance à des produits de filiation solides et radioactifs, en grande majorité à courte période (quelques minutes à quelques jours), à l'exception du plomb 210 (22,3 ans) et du polonium 210 (138 jours).

Les descendants solides du radon sont à l'origine d'une émission $\gamma$ intense, en particulier le plomb 214, et le bismuth 214, dont les raies étaient celles utilisées dans les traitements. Dans un objet, le radium 226 et ses descendants à vie courte se mettent en équilibre radioactif en quelques semaines, si le radon reste confiné.

\subsection{Les risques radiologiques du radium 226 et des $O R U M$}

Sur le plan de la radioprotection, la manipulation d'ORUM sans précaution particulière présente deux types de risque.

Le risque d'exposition externe : une aiguille de radium classique (activité égale à $37 \mathrm{MBq}$ ) donne un débit de dose absorbée de $3,3 \mathrm{mGy} / \mathrm{h}$ mesuré au contact d'une chambre d'ionisation de type Babyline ${ }^{\circledR}$, sous $300 \mathrm{mg} / \mathrm{cm}^{2}$. Or, il existe des objets dont l'activité peut atteindre $3700 \mathrm{MBq}$ (voir Sect. 8.4.2). La plupart du temps, une exposition de quelques dizaines de minutes à proximité de ces objets peut entraîner un dépassement de la limite maximale admissible pour le public, soit $1 \mathrm{mSv} / a n$ ainsi que des expositions localisées pouvant conduire à l'apparition d'effets déterministes. 
Le risque d'exposition interne par ingestion, inhalation ou contact par blessure: en cas de rupture de l'intégrité de l'objet, il peut y avoir une dissémination des sels de radium contenus à l'intérieur de l'objet détérioré. Ce risque de détérioration est surtout réel pour les plaques de Monel qui se fragilisent au cours du temps. Par contre, les descendants solides du radon peuvent parfois contaminer l'intérieur, voire l'extérieur des containers de stockage. Le radium 226 est classé parmi les isotopes de très forte radiotoxicité et celle des descendants du radon va d'une radiotoxicité très forte à modérée.

\subsection{Les risques liés aux conditions de détention}

Les situations les plus critiques se rencontrent chez les particuliers qui entreposent leurs sources dans une cave, un garage ou un grenier, mais parfois directement dans une pièce de vie de l'habitation, ou dans le jardin où elles subissent les intempéries. Dans certaines situations, les sources peuvent être disposées d'une manière telle que le public est susceptible d'être exposé.

Comme déjà souligné, les emballages d'origine sont sous-dimensionnés par rapport à la quantité de radium présente à l'intérieur. Les hôpitaux ou cliniques dotés d'un service de radiothérapie ou de médecine nucléaire et les centres anticancéreux disposent de locaux appropriés pour l'entreposage de produits radioactifs. En outre, la présence obligatoire dans l'établissement d'une personne compétente en radioprotection, qui est très souvent le radiophysicien du service de radiothérapie, renforcent les garanties concernant les conditions d'entreposage.

Dans tous les cas, ces entreposages doivent être considérés comme provisoires, car ils permettent rarement de maîtriser la totalité des risques, en particulier ceux liés à la contamination, au dégazage du radon, et à la perte de mémoire.

\subsection{La perception des risques liés à la détention d'objets au radium}

Aux risques précédemment rappelés, il convient d'ajouter celui, et ce n'est pas le moindre, découlant d'une perception sous-évaluée de ces risques. En effet, si certains praticiens ont manipulé ces objets avec des pinces à distance, d'autres les utilisaient durant toute leur vie à mains nues.

Ainsi, il n'est pas rare de voir d'anciens médecins manipuler encore aujourd'hui les containers d'origine à mains nues alors que parfois les débits de dose atteignent plusieurs milligrays par heure au contact.

De même, des particuliers ignorent complètement les conséquences d'une mauvaise manipulation, tout simplement par méconnaissance du risque des 
rayonnements ionisants. De plus, ces objets peuvent être entreposés depuis longtemps au même endroit, sans être à l'origine d'effets sur la santé ayant pour conséquence de banaliser le risque potentiel.

\section{La deuxième campagne de collecte des objets au radium}

\subsection{Pourquoi une seconde campagne de collecte?}

Ce radium contenu dans des objets désormais inutiles mais potentiellement dangereux n'a plus aucune valeur marchande. Il constitue donc un déchet radioactif qui doit être récupéré et mis en sécurité avant un stockage définitif.

Les récentes affaires de découvertes de sources de radium (en 1998, la découverte d'aiguilles de radium dans un cabinet de radiologie à Nice, en 1999 la clinique Condorcet à Lens, abandonnée et squattée depuis 10 ans et à Bruay-laBuissière des objets dans un abri de jardin situé à côté du mur mitoyen d'une école) ont montré encore l'existence d'ORUM qui n'étaient pas en sécurité et qui auraient pu être à l'origine de conséquences sanitaires pour les personnes exposées. Toute source de radium non-conditionnée correctement représente donc un danger potentiel pour la population.

De plus, l'ANDRA est saisie depuis quelques années par des personnes souhaitant se débarrasser de leurs sources, sans avoir de satisfaction à leur demande, du fait de l'absence de solution d'entreposage et de financement.

Pour ces diverses raisons, l'OPRI et l'ANDRA, à la demande du ministère chargé de la Santé, ont mis en place une nouvelle campagne de collecte.

\subsection{Organisation de la campagne}

L'organisation de la campagne de collecte est annoncée officiellement par le ministère chargé de la Santé, aux préfectures par la circulaire DGS/DH n 99/661 du 2 décembre 1999, qui précise par ailleurs les modalités pratiques de cette campagne et le rôle de chaque organisme. Les modalités opérationnelles ont été précisées dans une convention signée entre l'OPRI et l'ANDRA, le 17 novembre 1999.

Sur la base du volontariat, tout détenteur d'objet au radium à usage médical, peut contacter par un numéro vert, une cellule opérationnelle composée de représentants de l'OPRI et de l'ANDRA (voir Fig. 1). Cette cellule opérationnelle se charge de recueillir des informations précises de la part du détenteur. Elle vérifie d'une part, la cohérence des informations fournies afin de décider de la suite à donner et d'autre part, elle apprécie le degré d'urgence de la situation. 


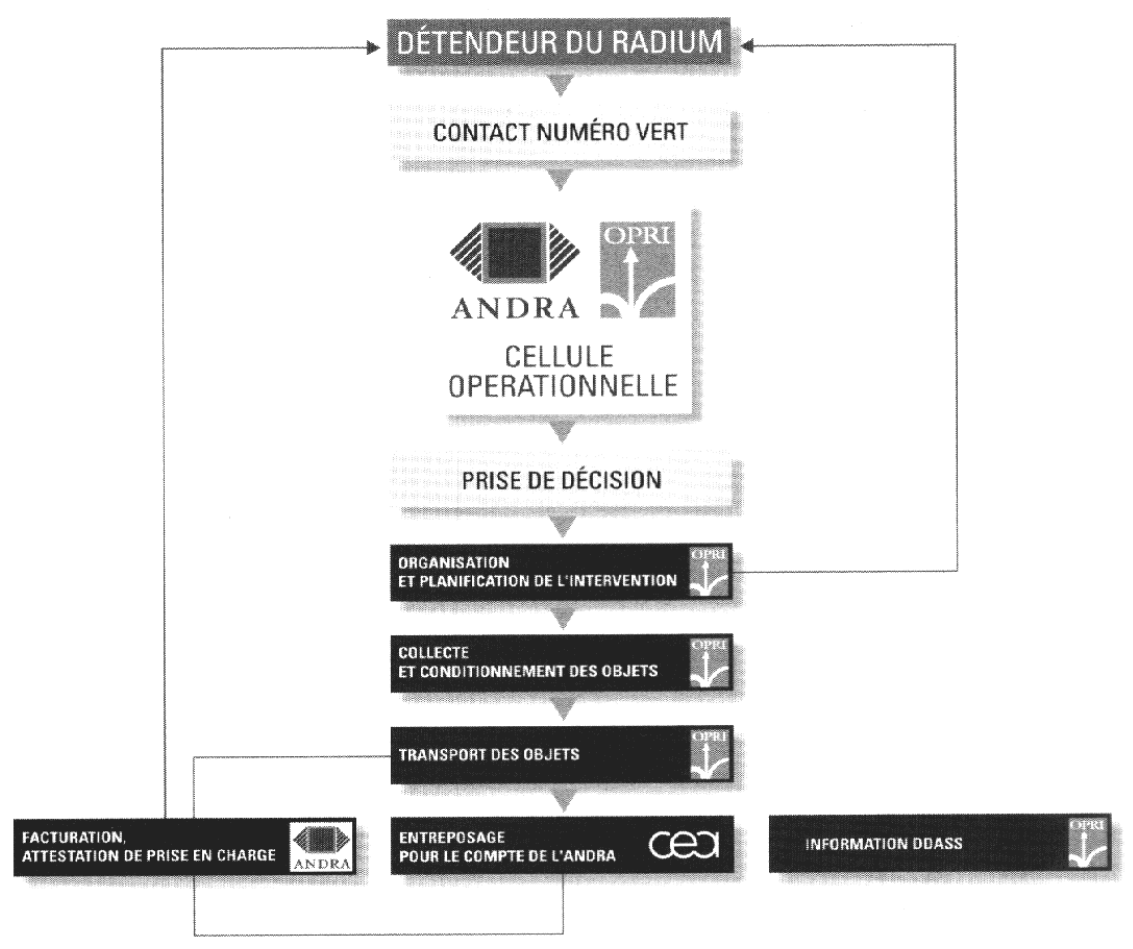

Figure 1 - Organisation chronologique d'une opération de collecte.

Chronology of a collection operation.

Dans le traitement des demandes, une priorité absolue est donnée aux particuliers pour les raisons invoquées précédemment. Une fois le dossier d'enlèvement constitué, l'ANDRA et l'OPRI agissent de manière autonome, en collaboration avec le CEA :

- l'OPRI planifie les interventions de collecte, assure le conditionnement et le transport des sources jusqu' au CEA de Saclay à l'INB 72, pour un entreposage en toute sécurité dans l'attente d'une solution pérenne pour leur stockage définitif par l'ANDRA. Par la suite, l'OPRI informe par courrier les DDASS en précisant les lieux de l'intervention, le nombre d'objets collectés et l'activité totale ;

- l'ANDRA, pour sa part, assure la facturation des opérations de collecte, délivre les attestations de prise en charge des déchets et gérera in fine le stock des colis conditionnés. Il est demandé une contribution financière de principe de $100 \mathrm{~F}$ quel que soit le nombre d'objets, qu'il s'agisse d'un particulier ou d'un professionnel. 


\section{Le déroulement de l'intervention de collecte}

L'intervention consiste à mettre en sécurité les objets au radium, dans des containers en plomb, agréés pour le transport de matières radioactives. En raison des risques liés à la manipulation de ces objets et des contraintes réglementaires, les intervenants appliquent un protocole strict, destiné à garantir leur sécurité ainsi que celle du public et à respecter la réglementation du transport de matières dangereuses.

\subsection{Les contraintes de l'intervention}

Ces contraintes, aussi bien d'ordre réglementaire que sanitaire, doivent être intégrées dans le déroulement des interventions, afin de garantir le respect des règles en vigueur, malgré les conditions d'intervention.

\subsubsection{Les risques liés à l'intervention}

Une intervention de collecte présente deux types de risque: des risques radiologiques liés à la manipulation de source de radium et des risques classiques dus à la manutention de charges lourdes (conteneurs et briques de plomb). Pour se prémunir de ces risques, les intervenants mettent une combinaison de travail, trois paires de gants jetables et des chaussures de sécurité. En cas de risque de contamination avéré, le port d'un masque est nécessaire. Par ailleurs, chaque intervenant est équipé d'un film dosimétrique, d'un dosimètre opérationnel et d'une bague thermoluminescente.

\subsubsection{La réglementation du transport de matière radioactive}

L'arrêté ADR du 5 décembre 1996, fixe des limites en débit de dose et en contamination surfacique des colis et des véhicules (voir Tab. II). Ces objets doivent être transportés dans des colis agréés type A ayant une certaine résistance mécanique. L'activité limite transportable est de $20 \mathrm{GBq}(500 \mathrm{mCi})$ pour le radium 226.

Ces colis sont constitués d'un tube en aluminium qui reçoit toutes les sources, d'un container de plomb d'épaisseur variable $(2,3$ ou $5 \mathrm{~cm})$, d'un calage en bois ou en carton et d'un emballage métallique ou cartonné. Par ailleurs le transport est effectué au moyen d'un véhicule spécifique, aménagé et équipé en conséquence.

\subsubsection{La protection du public}

Les interventions se déroulent soit dans un lieu accessible au public (hôpital, clinique, cabinet de radiologie...), soit directement chez le particulier. Dans les 
TABLEAU II

Réglementation du transport de matière radioactive. Transport regulation for radioactive materials.

\begin{tabular}{|c|c|c|c|}
\hline m+4. & $4+4$ & 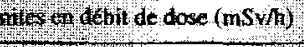 & Wty \\
\hline Golis. & W4t & W 1 mo & (t) \\
\hline Au contact & 2 & Contact des parois verticales & 2 \\
\hline \multirow[t]{2}{*}{ À I mètre } & 0,1 & À 2 mètres & 0,1 \\
\hline & & Intérieur cabine & 0,02 \\
\hline
\end{tabular}

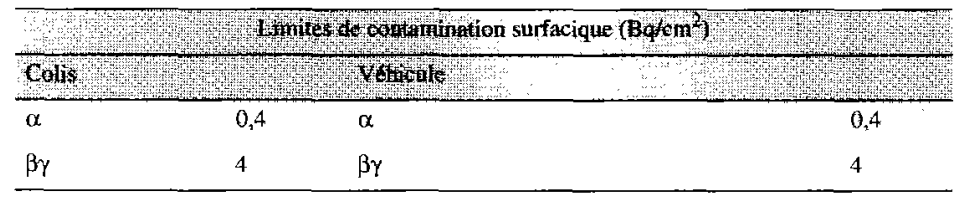

deux cas, il convient d'éviter toute exposition du public et toute contamination résiduelle consécutive à l'intervention.

\subsection{Le protocole d'intervention}

Les objectifs de ce protocole sont les suivants :

- définir les grandes lignes de l'organisation de la collecte,

- préciser la liste des équipements individuels de chaque intervenant,

- fixer la liste du matériel nécessaire au déroulement de l'intervention,

- organiser les opérations de conditionnement au moyen d'une procédure technique d'intervention.

\subsection{La procédure technique d'intervention}

Cette procédure décrit les principales étapes d'une opération de collecte. Il est bien entendu indispensable de l'adapter en fonction des conditions particulières. Une intervention peut être décomposée en succession de phases réparties selon le modèle suivant.

\section{$I^{\text {re }}$ phase : l'approche}

Cette phase d'observation et de réflexion est destinée, d'une part, à apprécier la difficulté de l'intervention et d'autre part, à effectuer des relevés radiamétriques, dans et autour de la zone d'entreposage des sources. Les intervenants s'habillent et mettent leurs équipements individuels de protection et leurs dosimètres. $\mathrm{Si}$ nécessaire un balisage est mis en place, pour assurer la radioprotection du public. 


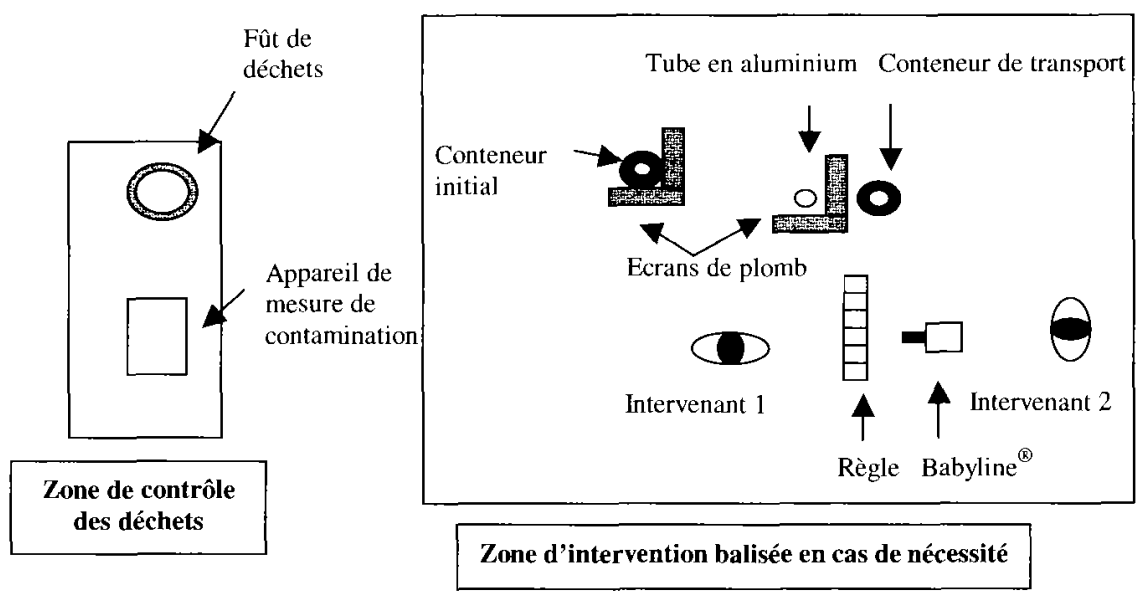

Figure 2 - Disposition de la zone de travail. Arrangement of work zone.

\section{$2^{e}$ phase : la mise en place de la zone de travail}

Elle constitue une phase préparatoire essentielle de l'intervention et conditionne son bon déroulement. Une zone mal disposée peut rendre l'intervention plus difficile et par conséquent augmenter l'exposition du personnel. En outre, elle doit être adaptée aux contraintes particulières liées à la configuration des lieux.

Cette zone comprend trois postes de travail aménagés pour éviter toute dispersion de contamination radioactive (voir Fig. 2): un poste de conditionnement des sources, constitué d'une zone de mesure pour estimer l'activité et d'une zone de conditionnement, un poste de contrôle et de conditionnement des déchets technologiques générés pendant l'intervention et une zone d'entreposage des colis conditionnés éloignée des intervenants afin d'éviter toute exposition inutile. Durant cette phase, les intervenants testent la gestuelle de l'intervention et la disposition de la zone de travail, afin d'adapter si nécessaire sa disposition.

\section{$3^{e}$ phase : le conditionnement des objets}

C'est par définition la phase la plus critique de l'opération car les sources sont sorties l'une après l'autre de leur emballage d'origine pour être mesurées et conditionnées. Au préalable des contrôles de contamination sont réalisés par frottis 
sur l'emballage (intérieur et extérieur). En cas de nécessité une spectrométrie $\gamma$ peut être faite in situ avec un appareil portable pour lever le doute. Tous les objets sont manipulés à l'aide d'une pince à distance : ainsi chaque source est transférée depuis l'emballage d'origine jusqu'au container de transport en mesurant le débit de dose absorbée au contact d'une chambre d'ionisation de type Babyline ${ }^{\circledR}$, en vue d'évaluer son activité.

$4^{e}$ phase : les contrôles

Des contrôles réglementaires des colis jusqu'au contrôle des intervenants, c'est une phase essentielle d'un point de vue sanitaire et réglementaire. Cinq types de contrôle sont effectués durant l'intervention :

- contrôle de contamination et des débits de dose au contact et à $1 \mathrm{~m}$ des colis avant étiquetage (réglementaire),

- contrôle de contamination du fût de déchets induits (réglementaire),

- contrôle de non-contamination du site de l'intervention (bonne pratique),

- contrôle du matériel et des intervenants (bonne pratique).

$5^{e}$ phase : le repli

L'ultime phase de l'intervention consiste aussi bien à ranger tout le matériel qu'à préparer le transport des colis vers le centre CEA de Saclay (rédaction des documents nécessaires, pose de la signalisation réglementaire sur le véhicule). Il est nécessaire d'optimiser la répartition des colis dans le véhicule et de disposer des protections biologiques afin de réduire au maximum le débit de dose dans la cabine.

\subsection{Les difficultés rencontrées au cours des opérations de collecte}

Au regard des conditions de déroulement des interventions, il n'est pas anormal que les équipes de l'OPRI rencontrent périodiquement des difficultés. L'objectif de cette partie ne consiste pas à dresser une liste exhaustive de toutes ces difficultés et des solutions mises en œuvre pour y remédier. Bien souvent les petits problèmes quotidiens trouvent une solution localement. Par contre il apparaît nécessaire d'en mentionner un certain nombre, récurrents, apparus à une période bien avancée du déroulement de la campagne et solutionnés non sans difficulté.

\subsubsection{L'entreposage temporaire des sources pendant la nuit}

En raison des risques liés au vol, au vandalisme ou à des actes de malveillance, il ne serait pas judicieux de laisser le chargement sans surveillance, pendant le repos des intervenants, lorsque les opérations se déroulent sur plusieurs jours. De ce fait, 
après chaque journée, les colis conditionnés sont laissés sur le lieu de la dernière intervention s'il s'agit d'un centre de soins disposant de structures permettant l'entreposage en toute sécurité. Dans le cas d'un particulier, l'OPRI sollicite la collaboration d'un centre de soins disposant de locaux adaptés. Il convient de remercier tous ces centres pour l'accueil réservé aux équipes d'interventions et l'aide qu'ils ont pu nous apporter.

\subsubsection{L'activité des sondes de Crowe}

La majorité des sondes de Crowe rencontrées a une activité avoisinant $3700 \mathrm{MBq}$, soit supérieure à celle initialement mentionnée dans le mémento de la collecte (ANDRA, 1998) (1 $850 \mathrm{MBq}$ ). Ainsi, les containers de $5 \mathrm{~cm}$ de plomb qui ne peuvent accepter que $1850 \mathrm{MBq}$, ne sont pas utilisables tels quels pour le transport. En effet, une telle activité ne permet pas le respect des normes de transport. Or, l'ANDRA ne dispose d'aucun container plus épais agréé pour le transport. Pour y remédier, $1 \mathrm{~cm}$ de plomb a été ajouté dans le tube en aluminium avec un trou intérieur de $3 \mathrm{~mm}$ destiné à recevoir la partie radioactive de la sonde de Crowe afin d'augmenter l'épaisseur totale du conteneur.

\subsubsection{Le dégazage du radon}

Le problème du dégazage de radon se pose essentiellement pour les sondes de Crowe. Lors du transport des sources ou dans l'attente de l'acheminement au CEA, il se produit des phénomènes de dégazage de radon, dont les descendants solides ont tendance à contaminer la surface extérieure du colis, le rendant ainsi non conforme aux règles du transport de matières dangereuses.

En collaboration avec le CEA, des solutions techniques ont été recherchées afin de confiner ce gaz. La première solution consiste à appliquer autour du bouchon du tube en aluminium une colle spécifique, étanche au gaz. La deuxième solution consiste à conditionner les objets dans des flacons de type pénicilline sertis permettant ainsi un confinement du gaz. Ces deux solutions ont été testées et ont permis d'éviter le développement de ces contaminations.

\subsubsection{Des témoignages des détenteurs souvent erronés}

D’une manière générale, il est possible de douter de chaque témoignage en raison d'un décalage entre ce qui est déclaré et ce qui est effectivement retrouvé sur place. La décision de réaliser une intervention est prise, dans la majorité des cas, sur la base des déclarations du détenteur. Très souvent, ce dernier déclare la présence d'objets au radium, sans en connaître précisément leur nature, leur nombre et leur activité. Parfois certains détenteurs sont en mesure d'annoncer la nature des objets sans pouvoir fournir d'indications complémentaires, et vice versa. 
Ces témoignages erronés trouvent leur origine dans une méconnaissance des objets détenus en raison de l'absence de toute réglementation vis-à-vis de la détention du radium et dans un manque de traçabilité de ces sources (très souvent absence de documents). De plus, dans la plupart des cas, elles ont été héritées et il s'agit souvent d'une découverte fortuite du détenteur.

L'inconvénient majeur de ce problème réside dans la préparation de l'intervention qui n'est pas facilitée, notamment en ce qui concerne la prévision du nombre et de la taille des conteneurs de transport. Afin de palier à toute éventualité, compte tenu du retour d'expérience des premières interventions, ce nombre est toujours majoré et les équipes de l'OPRI emportent plus de conteneurs que ce qui serait normalement nécessaire.

\section{La gestion des équipes d'intervention}

\subsection{La dosimétrie des intervenants}

Afin de limiter les doses individuelles, une contrainte de dose de $500 \mu \mathrm{Sv}$ a été fixée, pendant toute la durée de la campagne, pour un nombre d'interventions estimé à 12 par intervenant. Par ailleurs, tous les intervenants ont reçu au préalable une formation théorique et pratique spécifique leur permettant d'apprécier les différentes phases de l'intervention mais aussi d'apprendre les gestes de base pour travailler rapidement et efficacement. Ce personnel est bien entendu classé en catégorie A et il est suivi médicalement.

Compte tenu de sa fonction habituelle et de ses missions quotidiennes qui doivent être conciliées avec cette campagne chaque agent intervient à fréquence très variable. Ainsi, il existe deux catégories d'intervenants: les intervenants réguliers, pour lesquels la campagne fait partie de leurs attributions habituelles, et les suppléants qui participent ponctuellement aux interventions. Du fait de ces différences, on observe des écarts importants entre les doses individuelles reçues. Par ailleurs, en fonction de la difficulté de l'intervention, du nombre de colis conditionnés et du temps de trajet, la dose reçue varie d'une intervention à l'autre.

Sur la base de ces différents facteurs, les doses individuelles opérationnelles des 22 intervenants durant toute la campagne s'échelonnent entre 5 et $452 \mu \mathrm{Sv}$. La dose collective est proche de 3,1 homme. $\mathrm{mSv}$ pour l'ensemble des opérations soit un coût dosimétrique de $0,88 \mu \mathrm{Sv}$ pour $37 \mathrm{MBq}(1 \mathrm{mCi})$ de radium collecté.

En fin d'intervention une fiche de dosimétrie reprenant le nom des intervenants, le numéro de la bague thermoluminescente ainsi que les données de la dosimétrie opérationnelle est établie. Après l'intervention le responsable de la collecte au sein de l'OPRI assure le suivi de la dosimétrie opérationnelle collective et individuelle. 


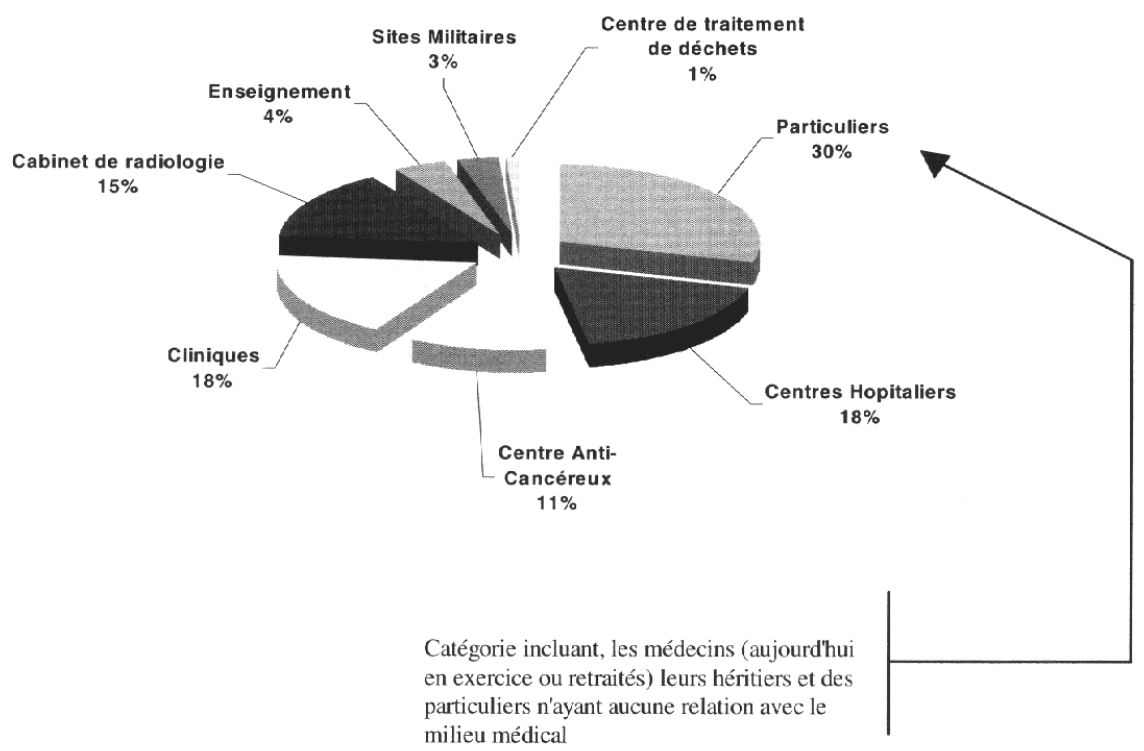

Figure 3 - Répartition des lieux d'intervention.

Types of collection sites.

\subsection{Gestion du personnel intervenant}

En général, les interventions mobilisent deux à trois intervenants suivant la difficulté prévisible de l'intervention. Selon la zone géographique concernée, les divisions régionales de l'OPRI peuvent servir d'appui technique avant et pendant l'intervention. Ainsi, avant, elles sont en mesure d'effectuer des opérations de contrôle afin de lever le doute dans le cas d'un témoignage douteux sur la présence d'ORUM ou de faire des premières mesures et une mise en sécurité des sources avant la collecte. Pendant l'intervention, les divisions régionales apportent leur concours technique en renforçant les équipes du Vésinet dans le déroulement des opérations.

\section{Bilan général de la collecte en quelques chiffres}

Durant l'ensemble de la campagne, l'OPRI (équipes du Vésinet et divisions régionales) a réalisé 90 interventions au cours desquelles 517 objets ont été collectés et conditionnés dans 133 colis. $14 \%$ de ces interventions sont des contrôles préalables avant enlèvement ou des opérations de lever de doute. La figure 3 présente la répartition des lieux d'intervention quel que soit le type d'intervention (lever de doute, contrôle préalable, collecte). 


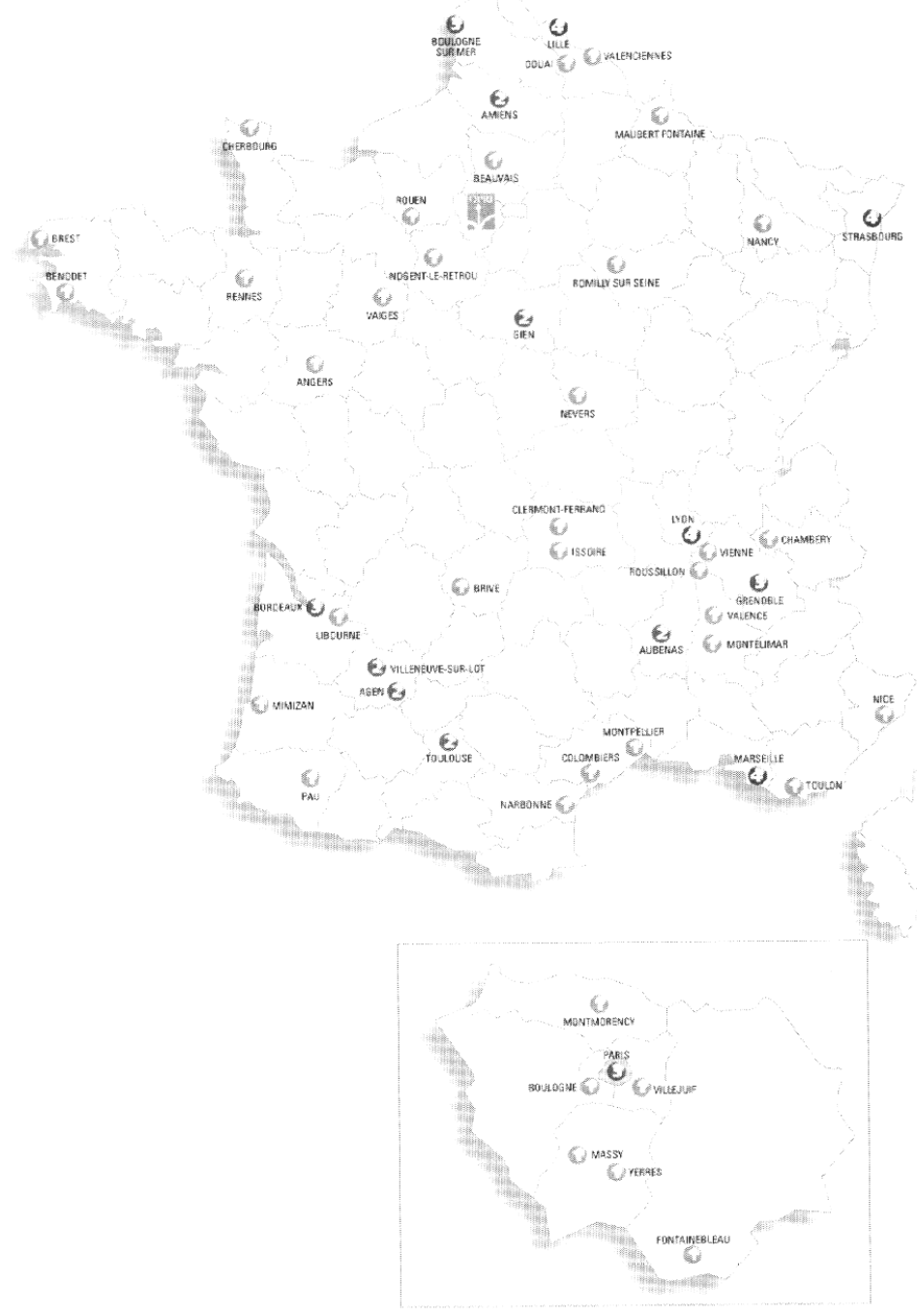

Figure 4 - Carte des interventions de collecte, réalisées par l'OPRI pendant la campagne. Map of collection operations carried out by OPRI during the campaign.

La figure 4 représente les lieux géographiques et le nombre d'interventions de collectes réalisées dans chaque agglomération. L'activité totale collectée pour l'ensemble des interventions est de $3,5 \mathrm{Ci}$ soit près de $1,32 \times 10^{11} \mathrm{~Bq}$. Le tableau III 


\section{TABLEAU III}

Bilan des objets collectés : période du $1^{\mathrm{er}}$ décembre 1999 au 31 octobre 2001. Number and activity of objects collected (Dec. 1999, 1 - Oct. 2001, 31).

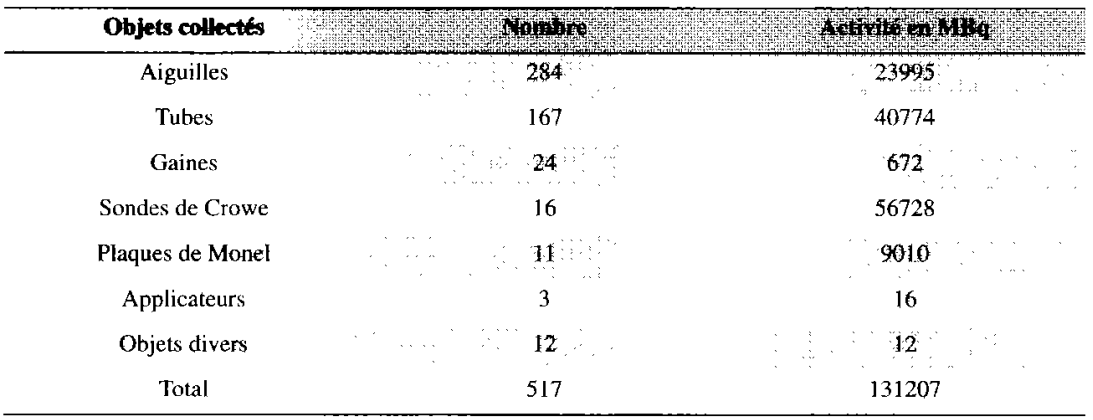

présente le bilan du nombre d'objets collectés ainsi que les activités totales estimées par type d'objet.

\section{Conclusion}

Cette campagne de collecte a montré tout son intérêt dans le domaine de la santé publique. C'est ainsi que des personnes du public ont été soustraites à des expositions inutiles et le plus souvent ignorées. En outre des sources en déshérence ont été récupérées et mises en sécurité. Par ailleurs, elle a permis de mettre également en évidence d'autres objets contenant du radium, comme les fontaines à radon ou des compresses considérées comme du matériel paramédical. Si le bilan de cette campagne est donc largement positif, néanmoins on ne peut pas considérer la tâche comme totalement accomplie. Dans ces conditions, l'OPRI pour sa part est prêt à poursuivre cette campagne, d'autant plus qu'elle présentait l'avantage de par son organisation de simplifier les démarches nécessaires à l'enlèvement et à la prise en charge de ces objets.

\section{RÉFÉRENCES}

ANDRA (1997) Inventaire national des déchets radioactifs, pp. B 1-B 18.

ANDRA (1998) Mémento pratique pour la collecte d'objets au radium utilisés en médecine dans les années 1920 à 1960, pp. 1-17.

Boudia S. (1997) L'industrie du radium et le laboratoire Curie : une histoire commune, Inventaire national des déchets radioactifs (ANDRA), pp. B19-B22

DGS/DH (1999) Circulaire $n^{\circ} 99 / 661$ du 2 décembre relative à la campagne nationale de collecte d’objets à usage médical contenant du radium.

Dutreix A., Marinello G., Wambersie A. (1982) Dosimétrie en curiethérapie. Principales sources utilisées en curiethérapie, pp. 31-33. Masson, Paris.

Tubiana M. (1999) Le centenaire de la découverte de la radioactivité, Lett. Cancér. Radioth. 8 (1), $9-11$. 